\title{
The Effect of Zingiber Officinale (Ginger) Extract on Blood Pressure and Heart Rate in Healthy Humans
}

\author{
L.S. Ojulari ${ }^{1}$, O.T. Olatubosun ${ }^{1}$, K.B. Okesina ${ }^{1}$, B.V. Owoyele ${ }^{1}$ \\ 1- Department of Physiology, Faculty of Basic Medical Sciences, College of Health Sciences, University of \\ Ilorin, Ilorin, Nigeria
}

\begin{abstract}
Zingiber officinale (ZO) has a long history of traditional use. It contains several constituents such as gingerol, gingerdiol, gingerdione, beta-carotene, capsaicin, caffeic acid and curumin. The present study was undertaken to investigate the effect of consumption of $\mathrm{ZO}$ on the heart rate and blood pressure (BP).

Subjects for the study comprised of 22 males and 38 females making a total of 60 subjects. The subjects were in 3 groups of 20 each; a Control group (distilled water) and 2 experimental groups (100mg/kg bodyweight and $50 \mathrm{mg} / \mathrm{kg}$ bodyweight of oral consumption of ZO extract). For each group, three readings were obtained per subject- one before administration, and the other two readings at intervals of $2 \mathrm{hrs}$ and 4 hrs after consumption of water or $Z O$. The readings taken comprise of systolic BP, diastolic BP, and the heart rate.

Results showed a significant $(p<0.05)$ decrease in all measured parameters $2 \mathrm{hrs}$ after $Z O$ administration and a significant ( $p<0.05)$ increase in only the systolic BP, 4hrs after ZO administration.

It can be concluded that consumption of ZO at doses employed in this study, has a blood pressure lowering effect and this effect is dose-dependent.
\end{abstract}

Keywords: Zingiber officinale, systolic, diastolic, blood pressure, heart rate

\section{Introduction}

The use of herbs or medicinal plants in the treatment of diseases worldwide dates back to pre-historic times. One of the earliest records of the use of herbal medicine is that of Chaulmoogra oil from species of Hydrocarpus gaerth, which was found to be effective in the treatment of leprosy.

Zingiber officinale (ZO), commonly known as "ginger", is indigenous to southern China, from whence it spread to the Spice Islands and other parts of Asia, and subsequently to West Africa and the Caribbean. Ginger has been used as dietary spice, as well as in traditional oriented medicine (Surh et al; 1999). Due to its acclaimed medicinal effect, many people now take it so well in different ways. Notable among the medicinal uses of $\mathrm{ZO}$ is in the treatment of digestive problems, such as nausea, indigestion, intestinal infection and flatulence. It has also been reported to cure motion sickness (Cared du; 1999). In Nigeria, there are increasing reports on the use of some local herbs on infections such as diabetes, hemorrhoids, small pox, hypertension, paralysis and dysentery (Abimbola; 1986).

ZO contains many cations and anions, among which are calcium, magnesium and phosphorus that function in bone formation, muscle contraction and nerve transmission. The high content of these minerals in ZO make it a useful candidate for muscle spasms, depression, hypertension, muscle weakness, convulsions, confusion, personality changes, nausea, lack of coordination and gastrointestinal disorders. The high content of potassium in ginger could protect the body against bone fragility, paralysis and sterility. In addition to the role of potassium in blood pressure regulation, it also regulates heartbeat (Shelly et al; 2004).

The present study was therefore undertaken to investigate the effect of consumption of $\mathrm{ZO}$ on the cardiovascular system (CVS), using changes in heart rate and blood pressure (BP) as the indices.

\section{Materials And Methods}

The study was a randomized safety trial comprised of 60 subjects, 22 males and 38 females, from the Faculty of Basic Medical Sciences. The subjects were in 3 groups of 20 each. Informed consent was given by the subjects and ethical clearance was obtained from the Research Ethic Committee, University of Ilorin.

\subsection{Study setting:}

The study was conducted on the $14^{\text {th }}$ of December, 2013 at The University of Ilorin, Kwara state, Nigeria.

\subsection{Extract preparation and administration:}

$250 \mathrm{gms}$ of rhizomes of ZO was brought from Oja Oba market in Ilorin, Nigeria. The covers were peeled off and the peeled rhizomes washed thoroughly with cold water and allowed to dry. The dried rhizomes were the powdered mechanically, thus made ready for extraction. The product was the transferred to the central 
tubule of the soxhlet apparatus. The filtrate was concentrated to dryness by using a water bath and a yield of $9.6 \%(\mathrm{w} / \mathrm{w})$ was obtained.

There was a control group and two experimental groups. The control group consumed ordinary water (placebo), while the second and third groups consumed $\mathrm{ZO}$ at $100 \mathrm{mg} / \mathrm{kg}$ of bodyweight and $50 \mathrm{mg} / \mathrm{kg}$ of bodyweight, respectively.

\subsection{Experimental Groups:}

Group A- distilled water

Group B- $100 \mathrm{mg} / \mathrm{kg}$ bodyweight of ZO

Group C- $50 \mathrm{mg} / \mathrm{kg}$ bodyweight of ZO

There was a control group and two experimental groups. The Control group consumed ordinary water (placebo), while the second and third groups consumed $\mathrm{ZO}$ at $100 \mathrm{mg} / \mathrm{kg}$ of bodyweight and $50 \mathrm{mg} / \mathrm{kg}$ of bodyweight, respectively.

\subsection{Selection criteria:}

The following criteria were satisfied by each of the selected candidate:

- No history of cardio-pulmonary disease

- Availability and capacity to cooperate adequately during the study

- Not on any drug medication

- No exercise $36 \mathrm{hrs}$ prior to ZO extract administration

\subsection{Ethics:}

The ethics committee of The University of Ilorin approved the protocol. Written informed consent was obtained from the participants after comprehensive explanation of the procedure involved.

\subsection{Measurement of heart rate and blood pressure:}

The blood pressure was measured using a digital sphygmomanometer (OMRON Health Care, Europe B.V.) and the heart rate was also measured digitally using a digital pulse monitor (OMRON Health Care, Europe B.V.).

\subsection{Statistical Analysis:}

The mean values of the measured variables were determined and standard error of mean (SEM) were calculated. The test of significance was determined by using the Student's t-test. Differences in means were considered significant at $\mathrm{p}<0.05$.

\section{Results}

\subsection{The effect of $Z O(100 \mathrm{mg} / \mathrm{kg}$ bodyweight $)$ on cardiovascular responses:}

$100 \mathrm{mg} / \mathrm{kg}$ of bodyweight of $\mathrm{ZO}$ had a significant effect $(\mathrm{p}<0.05)$ on both the systolic and diastolic blood pressure, as well as the heart rate. After two hours of administration, the systolic BP was reduced from $114.3 \pm 3.22 \mathrm{mmHg}$ to $105.5 \pm 3.13$. The diastolic BP was also reduced from $73.3 \pm 3.35 \mathrm{mmHg}$ to $70.5 \pm 3.39$ mmHg. Likewise, the heart rate was significantly $(\mathrm{p}<0.05)$ reduced from $78.8 \pm 2.47$ beats/min to $70.2 \pm 2.55$ beats/min.

After $4 \mathrm{hrs}$, the systolic BP still remained significantly reduced $(\mathrm{p}<0.05)$ at $107.6 \pm 2.81 \mathrm{mmHg}$. The diastolic $\mathrm{BP}$ and the heart rate were not significantly altered after $4 \mathrm{hrs}$ of $\mathrm{ZO}$ administration when compared to control. This is shown in Table 1 below.

\subsection{Effect of $\mathrm{ZO}(50 \mathrm{mg} / \mathrm{kg}$ of bodyweight $)$ on cardiovascular responses:}

$50 \mathrm{mg} / \mathrm{kg}$ dose of $\mathrm{ZO}$ caused a significant $(\mathrm{p}<0.05)$ reduction in systolic BP after $4 \mathrm{hrs}$, but had no significant effect on the diastolic BP and the heart rate after 2 and 4 hrs. This is shown in Table 2 below.

\section{Tables}

Table 1: Effect of ZO (100mg/kg bw) on systolic BP, diastolic BP and heart rate

\begin{tabular}{|l|l|l|l|}
\hline Variables & Control (before administration) & 2hrs after administration & 4hrs after administration \\
\hline Systolic BP $(\mathrm{mmHg})$ & $114.3 \pm 3.22$ & $105 \pm 3.13^{*}$ & $107 \pm 2.81^{*}$ \\
\hline Diastolic BP (mmHg) & $73.3 \pm 3.39$ & $70.0 \pm 3.39^{*}$ & $70.4 \pm 2.38$ \\
\hline Heart rate (beats/min) & $78.8 \pm 2.47$ & $73.2 \pm 2.15^{*}$ & $80.0 \pm 3.07$ \\
\hline
\end{tabular}

* $-\mathrm{p}<0.05$ when compared to Control, ZO-Zingiber officinale, bw-bodyweight, BP-blood pressure 
The Effect of Zingiber Officinale (Ginger) Extract on Blood Pressure and Heart Rate in ....

Table 2: Effect of oral administration of $\mathrm{ZO}$ extract $(50 \mathrm{mg} / \mathrm{kg}$ bw) on systolic BP, diastolic BP and heart rate

\begin{tabular}{|l|l|l|l|}
\hline Variables & Control (before administration) & 2hrs after administration & 4hrs after administration \\
\hline Systolic BP $(\mathrm{mmHg})$ & $108.4 \pm 2.66$ & $107.5 \pm 2.02$ & $104 \pm 2.19$ \\
\hline Diastolic BP $(\mathrm{mmHg})$ & $66.2 \pm 1.65$ & $67.6 \pm 1.89$ & $65.7 \pm 1.61$ \\
\hline Heart rate (beats/min) & $77.6 \pm 2.20$ & $73.2 \pm 2.15$ & $74.3 \pm 2.36$ \\
\hline
\end{tabular}

ZO-Zingiber officinale, bw-bodyweight, BP-blood pressure

\section{Discussion}

In the present study, the hypotensive effect of ZO has been established. The study was broadened by the use of two different doses of $\mathrm{ZO}$.

ZO has a long history of traditional use. It contains several constituents such as gingerol, gingerdiol, gingerdione, beta-carotene, capsaicin, caffeic acid and curumin (Kikuzaki \& Nakatani; 1996, Schulick; 1996). This study showed a significant decrease in the systolic BP $(\mathrm{p}<0.05)$, after $2 \mathrm{hrs}$ and $4 \mathrm{hrs}$ of consumption of $100 \mathrm{mg} / \mathrm{kg}$ bodyweight of ZO. This dose also caused a significant $(\mathrm{p}<0.05)$ decrease in the diastolic BP and heart rate, but only after $2 \mathrm{hrs}$, and not at $4 \mathrm{hrs}$. This corresponds to findings by Suekawa (1984) and Srivastava (1984) who reported significant hypotension and bradycardia in rats following administration of ZO. On the other hand, consumption of $50 \mathrm{mg} / \mathrm{kg}$ bodyweight only caused a significant decrease in the systolic BP after 4 hrs while the heart rate and diastolic BP were not significantly altered following administration of this dose.

According to Srivastava, (1984), the fact that ZO consumption can lead to an increase in blood flow to the periphery of blood vessels indicates that the resistance of blood has been partially overcome. So by implication, ZO reduces total peripheral resistance. Since arterial BP is equal to the product of the cardiac output and total peripheral resistance (Egan \& Stammered; 1988), a reduction in total peripheral resistance will result in a decrease of arterial BP.

Fugh-Berman (2000), suggested that data from his study indicated that ZO's BP lowering effect was mediated through the blockade of voltage-dependent calcium channels. Another suggested mechanism may involve the serotonergic antagonistic property of ZO (Ghayur \& Ghilani; 2005).

\section{Conclusion}

In conclusion, human trials for the hypotensive effects of $\mathrm{ZO}$ have been few and generally used on low dose with inconclusive results (Nicroll \& Tenien; 2009). Our study conclusively showed that consumption of $\mathrm{ZO}$ at doses employed in this study, has a blood pressure lowering effect and that this effect is dose-dependent.

\section{References}

[1]. Y.J. Surh, K.K. Park, K.S. Chun, E. Lee, S.S. Lee, Antitumor-promoting activities of selected pungent phenolic substances present in ginger, Journal of Environmental Pathology, Toxicology and Oncology, 18(2), 1999, 131-9

[2]. P. Cared du, Motion sickness in children: results of a double blind study with ginger and dimenhydrinate. Health notes Review, 6 , 1996, 102-7

[3]. S.Abimbla, Traditional treatment for hypertension, stroke, asthma, sickle cell and diabetes in the state of medicinal plant research in Nigeria, Modern University Press pp, 1986, 125-133

[4]. T.E. Shelly, D.O. Melnnis, E. Pahio, J. Edu, Aromatherapy in the Mediterranean fruit fly (Diptera Tephritidae): Sterile males exposed to ginger root oil in pre-release storage boxes display increased mating competitiveness in freed-cage trials, Journal of Economic Entomology, 97(3), 2004, 846-53

[5]. H. Kikuzaki, N. Nakatani, Cyclic diarylheptanoids from rhizomes of Zingiber officinal, Phytochemistry, 43, 1996, 273-77

[6]. P. Schulick, Ginger, common spice and wonder drug, $3^{\text {rd }}$ edition. Brattleboro (VT): Herbal Free Press Ltd, 1996

[7]. M. Suekawa, A. Ishiage, K.Yuasa, Pharmacological studies on ginger pharmacological actions of pungent constituents, 6 -gingerol and 6-shogaol, Journal of Pharamcology and biodynamic, 7, 1984, 836-48

[8]. K.C. Srivastava, Effect of aqueous extracts of onion, garlic and ginger on platelet aggregation and metabolism of arachidonic acid in the blood vascular system, Prostaglandins, Leukotrienes and Medicine, 13, 1984, 227-35

[9]. B. Egan, R. Schmouder, The importance of hemodynamic considerations in essential hypertension, American Heart Journal, 116(2), 594-599

[10]. A. Fugh-Berman, Dietary supplements in the prevention and treatment of cardiovascular disease, Preventive Cardiology, 2000, 2432

[11]. M. N. Ghayur, A.H. Gilani, Ginger lowers blood pressure through blockade of voltage dependent calcium channels, Journal of Cardiovascular Pharmacology, 45, 2005, 74-80

[12]. R. Nicroll, M.Y. Henein, Ginger (Zinger offiicinale Roscoe): a hot remedy for cardiovascular disease? International Journal of Cardiology, 131, 2009, 408-9 